\title{
A novel torsional exciter for modal vibration testing of large rotating machinery
}

\author{
C. Sihler \\ MAX-PLANCK-INSTITUT FÜR PLASMAPHYSIK (IPP) \\ EURATOM Association \\ Boltzmannstr. 2, D-85748 Garching, Germany \\ Phone: +49 89 3299-1386, Fax: -2580 \\ E-mail: sihler@ipp.mpg.de
}

\begin{abstract}
A novel exciter for applying a dynamic torsional force to a rotating structure is presented in this paper. It has been developed at IPP in order to perform vibration tests with shaft assemblies of large flywheel generators (synchronous machines). The electromagnetic exciter (shaker) needs no fixture to the rotating shaft because the torque is applied by means of the stator winding of an electrical machine. Therefore, the exciter can most easily be applied in cases where a three-phase electrical machine (a motor or generator) is part of the shaft assembly. The oscillating power for the shaker is generated in a separate current-controlled DC circuit with an inductor acting as a buffer storage of magnetic energy. An AC component with adjustable frequency is superimposed on the inductor current in order to generate pulsating torques acting on the rotating shaft with the desired waveform and frequency. Since this torsional exciter does not require an external power source, can easily be installed (without contact to the rotating structure) and provides dynamic torsional forces which are sufficient for multi-megawatt applications, it is best suited for on-site tests of large rotating machinery.
\end{abstract}

Torsional exciter, electromagnetic shaker, rotordynamics, mechanical testing, torsional vibration, modal analysis

\section{INTRODUCTION}

Electromagnetic exciters for testing vibration properties of rotating structures were already proposed sixty years ago [1]. The first electromagnetic exciters were mainly developed for testing propellers without the necessity of using aircraft engines and to provide a vibration generator which simulates the rotary and vibratory stress of normal operating conditions while permitting a more satisfactory control or variation of the vibratory stress in accordance with different desired test conditions. The vibration generator consisted of a single-phase alternating-current motor, thus utilizing the characteristic of this type motor to reduce the motor torque to zero twice within each cycle of the alternating load current. The application of motor torque pulsations in single-phase motors was also proposed in experimental procedures for investigating torsional vibration in small air-conditioning units [2]. In this test procedure the fan motor was set up to act as a torsional exciter. This solution is attractive because modal vibration tests can be performed on site, without having to install an additional torsional exciter, e. g. a hydraulic exciter system [3]. Since both proposed electromagnetic exciters utilize the torque characteristic of a single-phase induction machine, they are not suited for an application to large electrical machinery which generally requires the application of three-phase machines. In three-phase electrical machines at balanced operation, the instantaneous power at each winding connected to the phases R, S, and T can be described by Equation System (1). The underlined parts of the equation do not contribute to the resulting torque transferred to the rotor. Therefore, the total active power transmitted by a three-phase electrical machine does not have a pulsating torque characteristic like a single-phase induction machine so that the test procedures proposed in [1] and [2] cannot be applied.

$$
\begin{aligned}
\begin{aligned}
P_{R}(t)= & u_{R}(t) \cdot i_{R}(t)=\hat{u} \cos (\omega t+\varphi) \cdot \hat{i} \cos \omega t \\
& =\frac{\hat{u} \cdot \hat{i}}{2}[\cos \varphi(1+\underline{\cos 2 \omega t)-\sin \varphi \sin 2 \omega t]}
\end{aligned} \\
P_{S}(t)=u_{S}(t) \cdot i_{S}(t)=\hat{u} \cos \left(\omega t+\frac{2 \pi}{3}+\varphi\right) \cdot \hat{i} \cos \left(\omega t+\frac{2 \pi}{3}\right) \\
\left.=\frac{\hat{u} \cdot \hat{i}}{2}\left[\cos \varphi\left[1+\frac{\left.\left.\cos 2\left(\omega t+\frac{2 \pi}{3}\right)\right]-\sin \varphi \sin 2\left(\omega t+\frac{2 \pi}{3}\right)\right]}{4 \pi}\right)\right]-\sin \varphi \sin 2\left(\omega t+\frac{4 \pi}{3}\right)\right]
\end{aligned}
$$

where

$$
\begin{array}{ll}
P_{R S T}: & \text { Instantaneous power at each winding } \\
u_{R S T}: & \text { Winding voltage } \\
i_{R S T}: & \text { Winding current } \\
\omega: & \text { Angular network frequency } \\
\varphi: & \text { Phase shift between } u_{R S T} \text { and } i_{R S T}
\end{array}
$$

The study and test of the dynamics of systems in which at least one part rotates with significant angular momentum is most relevant for large rotating machinery [4]. Testing of large rotating components poses significant practical difficulties in measurements and excitations. The different excitation techniques fall in two basic classes - transient excitation (free vibration) and continuous excitation (forced vibration). Since the rotating motion of the impact site is likely to cause errors in the measurement of frequency response functions [5], a harmonic (continuous) excitation device is preferable for testing large or complex rotating structures. In cases where a hydraulic torsional exciter cannot be applied, an electromagnetic exciter system which is capable of applying a sufficiently high dynamic torsional force to the 
rotating structure must be utilized. Such systems have been specially developed for laboratory tests of large rotating machinery [5]. They require the use of sufficiently large electro-magnets which are able to provide relevant dynamic forces to the rotating structure and have the disadvantage that they are difficult to move. Therefore, such exciter systems are not suited for on-site tests of large rotor shaft systems.

But in many cases on-site tests of large rotating machinery could easily be performed with a device similar to the one applied at IPP. Three-phase machines can provide an oscillating torque to a shaft system if the load current is modulated simultaneously in all three phases with an angular frequency $\Omega$ and an amplitude $m \cdot \hat{l}$ $(0<m<1)$. In modulating the stator current the instantaneous power at each stator winding is given by Equation System (2) assuming balanced operation [6].

$$
\begin{gathered}
P_{R}(t)=u_{R}(t) \cdot i_{R}(t)=\hat{u} \cos (\omega t+\varphi) \cdot \hat{i}\left[1+m \cos \left(\Omega t+\phi_{0}\right)\right] \cos \omega t \\
=\frac{\hat{u} \cdot \hat{i}}{2}\left[1+m \cos \left(\Omega t+\phi_{0}\right)\right] \cdot[\cos \varphi(1+ \\
\quad \frac{\cos 2 \omega t)-\sin \varphi \sin 2 \omega t]}{2} \\
P_{S}(t)=\frac{\hat{u} \cdot \hat{i}}{2}\left[1+\frac{\left.m \cos \left(\Omega t+\phi_{0}\right)\right] \cdot[\cos \varphi[1+}{\left.\left.\cos 2\left(\omega t+\frac{2 \pi}{3}\right)\right]-\sin \varphi \sin 2\left(\omega t+\frac{2 \pi}{3}\right)\right]}\right. \\
P_{T}(t)=\frac{\hat{u} \cdot \hat{i}}{2}\left[1+\frac{\left.m \cos \left(\Omega t+\phi_{0}\right)\right] \cdot[\cos \varphi[1+}{\left.\left.\cos 2\left(\omega t+\frac{4 \pi}{3}\right)\right]-\sin \varphi \sin 2\left(\omega t+\frac{4 \pi}{3}\right)\right]}\right.
\end{gathered}
$$

where

$\Omega: \quad$ Angular frequency of current modulation

$m \cdot \hat{\imath}$ : Amplitude of current modulation

$\phi_{0}$ : $\quad$ Phase angle of current modulation

If a stator current modulation can be achieved by means of a suitable device connected to the three-phase system of the electrical machine, a torque oscillating with the modulation frequency $\Omega$ is applied to the rotor. Since the oscillating torque is transferred with the same phase angle by all windings, its frequency $\Omega$ does not depend on the number of pole pairs of the electrical machine. The nominal power of such a device can be one or two magnitudes smaller than the nominal power of the electrical machine. This rating is sufficient for most experimental procedures involving large rotating machinery and enables a compact design of the device, so that it can be used for vibration tests on site.

Such tests had to be performed at IPP because of problems with torsional resonances which appeared in the shaft assemblies of large flywheel generators. Having investigated different methods of reducing the torsional stress in the generator shafts by simulation and measurement, IPP decided to solve these problems in developing an active damping device based on a feedback controlled DC circuit [7]. In 2003, one feedback controlled damping circuit was installed at each generator, therefore allowing one mode of torsional resonance to be damped efficiently. Testing and commissioning these active damping circuits required an experimental set-up enabling a continuous controlled excitation of torsional vibrations in the rotor shaft systems of the synchronous generators.

Section II describes this experimental set-up which enables a controlled excitation of torsional vibrations in the IPP flywheel generator shaft assemblies. After a discussion of typical simulation and measurement results in Section III, Section IV describes a compact torsional exciter which can easily be applied in modal vibration tests on site. After presenting results which can be achieved with such a device, the paper ends with a conclusion on this torsional excitation method.

\section{DESIGN OF THE IPP EXPERIMENTAL SET-UP FOR THE EXCITATION OF TORSIONAL VIBRATIONS}

Fig. 1 shows the IPP test set-up for the excitation of torsional oscillations in the shaft system of flywheel generator EZ3 (144 MVA / $500 \mathrm{MWs),} \mathrm{a} \mathrm{synchronous}$ generator with a shaft assembly weighing more than 100 tons. The "disturbance converter" in the test set-up is a current-controlled 6-pulse thyristor converter in bridge connection feeding an inductor (ASDEX Upgrade magnet

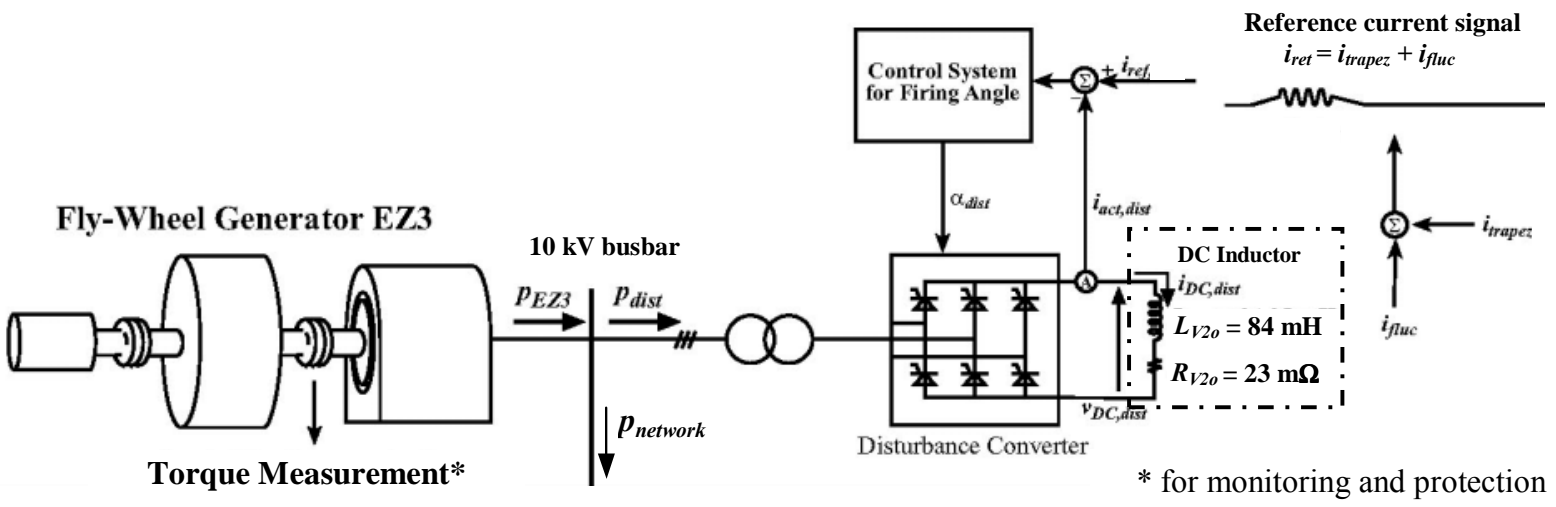

Fig. 1. Experimental set-up: Excitation of torsional (subsynchronous) oscillations in the shaft assembly of generator EZ3 
coil) with an inductance value of $84 \mathrm{mH}$ and an ohmic resistance of $23 \mathrm{~m} \Omega$. The converter output current was controlled in such a way that torsional oscillations were excited in the EZ3 shaft assembly. This can be achieved in supplying the inductor with a current consisting of a DC component ( $i_{\text {trapez }}$ in Fig. 1) and an AC component $\left(i_{f l u c}\right)$. The DC component is important because it enables the inductor in the DC circuit to be operated as a buffer storage of magnetic energy, being loaded and unloaded with an alternating current $i_{\text {fluc }}$. Thus an oscillating active power with a single, electronically adjustable frequency $\Omega$ can be generated if appropriate design values for the single components, especially the inductor, are chosen. In the general case the instantaneous power at the inductor terminals $P_{L}(t)$ can be calculated by Equation 3 .

$$
\begin{aligned}
P_{L}(t)= & u_{L}(t) \cdot i_{L}(t)=\left(I_{D C}+\hat{i}_{L} \cos \Omega t\right) \cdot\left(U_{D C}+\hat{u}_{L} \cos \left(\Omega t+\varphi_{L}\right)\right) \\
\Leftrightarrow P_{L}(t) & =\frac{\hat{u}_{L} \cdot \hat{i}_{L}}{2} \cdot \cos \varphi_{L}+U_{D C} \cdot I_{D C} \\
& +\frac{\hat{u}_{L} \cdot \hat{i}_{L}}{2} \cdot\left(\cos \varphi_{L} \cdot \cos 2 \Omega t-\sin \varphi_{L} \cdot \sin 2 \Omega t\right) \\
& +I_{D C} \cdot \hat{u}_{L} \cdot \cos \left(\Omega t+\varphi_{L}\right)+U_{D C} \cdot \hat{i}_{L} \cos \Omega t
\end{aligned}
$$

where

$$
\begin{array}{ll}
P_{L}: & \text { Instantaneous power at the inductor terminals } \\
u_{L}: & \text { Voltage at DC inductor, } \\
i_{L}: & \text { Current in DC inductor, } \\
I_{D C}: & \text { DC component of } i_{L}, \\
U_{D C}: & \text { DC component of } u_{L}, \\
\hat{u}_{L}: & \text { Amplitude of AC component of } u_{L}, \\
\hat{\imath}_{L}: & \text { Amplitude of AC component of } i_{L}, \\
\varphi_{L}: & \text { Phase shift between } u_{L} \text { and } i_{L} \\
\Omega: & \text { Angular frequency of AC component of } i_{L} \\
& \text { (identical to angular frequency in eq. (2)) }
\end{array}
$$

The frequency $\Omega$ and the DC and AC components of $i_{L}$ correspond to the reference current signal of the currentcontrolled thyristor converter. In choosing a DC inductor with high inductance and negligible ohmic resistance (which is the case in Fig. 1) and applying a reference current $I_{D C}>\hat{\imath}_{L}$, Equation (3) can be simplified as follows:

$$
\begin{aligned}
& \underline{P_{L}(t) \approx} \quad \frac{\hat{u}_{L} \cdot \hat{i}_{L}}{2} \cdot \cos \varphi_{L}+U_{D C} \cdot I_{D C} \\
&+I_{D C} \cdot \hat{u}_{L} \cdot \cos \left(\Omega t+\varphi_{L}\right) \\
& \approx \quad I_{D C} \cdot \hat{u}_{L} \cdot \cos \left(\Omega t+\varphi_{L}\right) \approx I_{D C} \cdot u_{L}(t)
\end{aligned}
$$

Considering only the fundamental component of the current input of the line-commutated thyristor converter feeding the DC inductor, the apparent power input can be calculated as given in Equation 5 [8].

where

$$
S_{1}=U_{d i 0} \cdot I_{D C}
$$

$S_{l}$ : $\quad$ Fundamental apparent input power

\section{$U_{d i 0}: \quad$ Converter no-load voltage}

The fundamental active power of a thyristor converter feeding a DC inductor can be calculated as indicated in Equation 6 [8]:

$$
\begin{aligned}
& P_{1}=S_{1} \cdot \cos \varphi_{1} \\
& P_{l}(t)=U_{d i \alpha}(t) I_{D C}=\left(U_{d i 0} \cos \alpha(t)\right) I_{D C}
\end{aligned}
$$

where

$P_{l}$ : $\quad$ Fundamental active input power

$\varphi_{1}$ : Phase shift angle of fundamental component

$U_{d i \alpha}$ : Converter output voltage

$\alpha$. Delay angle of thyristor converter

I. e. the fundamental component of the active input power of a line-commutated converter feeding a suitable DC inductor is directly proportional to the voltage modulation $U_{\text {dio }}(\mathrm{t})$. In choosing design values as given in Fig. 1, the fundamental active input power of a thyristor converter $P_{l}$ is equal to the instantaneous power at the DC inductor terminals $P_{L}$ and can be calculated by Equation (4). In using a six-pulse line commutated converter, current harmonics below the $5^{\text {th }}$ harmonic oscillation (corresponding to $250 \mathrm{~Hz}$ in a $50 \mathrm{~Hz}$-network) can be neglected. Therefore, the active power generated by a suitable thyristor-controlled torsional exciter features only one distinct frequency $\Omega$, higher harmonics only exist at a frequency greater than or equal to $5 \omega$.

\section{RESULTS OF SIMULATIONS AND TESTS BASED ON THE IPP EXPERIMENTAL SET-UP}

\section{A. Mechanical properties of the IPP flywheel generator shaft systems}

In the IPP experimental set-up the EZ3 generator was excited with a frequency $\Omega$ corresponding to the first natural frequency of the EZ3 shaft system which is $24 \mathrm{~Hz}$. This mechanical resonant frequency was estimated by means of the simple mass-spring model of the shaft assembly which is shown in Fig. 2 [7]. For high-speed rotors or the rotor system of a large flywheel generator, the kinetic energy of rotation can be a potential safety hazard. There may be mechanisms which transfer some of the rotational energy of the rotor into internal vibration modes of the machine [4]. The amount of kinetic energy

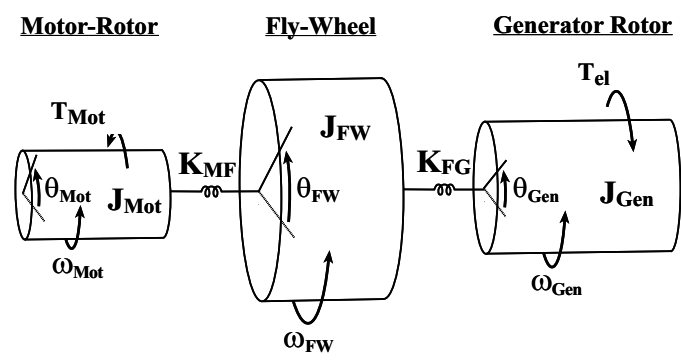

Figure 2. Mass-spring model of EZ3 shaft system 
available in the rotor is usually orders of magnitude greater than the deformational energy which any internal mode can absorb. Especially low torsional modes of a rigid rotor shaft system are characterized by very low natural damping values. The dynamic behaviour of a rigid rotor shaft system can be described by a partial differential equation system or, after a discretization by means of $\mathrm{n}$ finite elements, by the $\mathrm{n}$-dimensional differential equation system given in (7).

$$
J \ddot{\varphi}+D \dot{\varphi}+K \varphi=B v
$$

where

$\begin{array}{ll}\boldsymbol{\phi}: & \text { Vector of shaft torsion angles; } \\ \boldsymbol{v}: & \text { Vector of externally applied torques; } \\ \boldsymbol{J}: & \text { Matrix of moments of inertia; } \\ \boldsymbol{D}: & \text { Damping matrix; } \\ \boldsymbol{K}: & \text { Stiffness matrix; } \\ \boldsymbol{B}: & \text { Input matrix for external torques. }\end{array}$

Because of potential safety hazards in performing modal vibration tests at low torsional modes, the torsional exciter used in these tests must be capable of supplying an external torque not only with an adjustable frequency, but also with an adjustable amplitude. In performing tests with shafts of large diameter as they are used in the shaft system of turbine generators or large flywheel generators, small torsional angles in the range of tenths of a degree can correspond to torque values in the range of Meganewtonmeters (MNm). This requires the application of a suitable measurement and protection system. At IPP, a direct torque measurement based on an inductive sensor operating without contact was chosen [9]. With such a sensor the torque can be measured with high accuracy and low operational effort because the sensor can be installed at a shaft location where the torsional mode is well observable while the rotating machinery is in operation. The estimated first natural frequency of the EZ3 shaft system was confirmed in performing an FFT transformation of the signal from a torque measurement, as shown in Fig. 3. The measurement was performed during the swing-out phase of a torsional oscillation after a continuous excitation with the set-up shown in Fig. 1. During the measurement the sensor was installed at the coupling between the generator rotor and the flywheel.

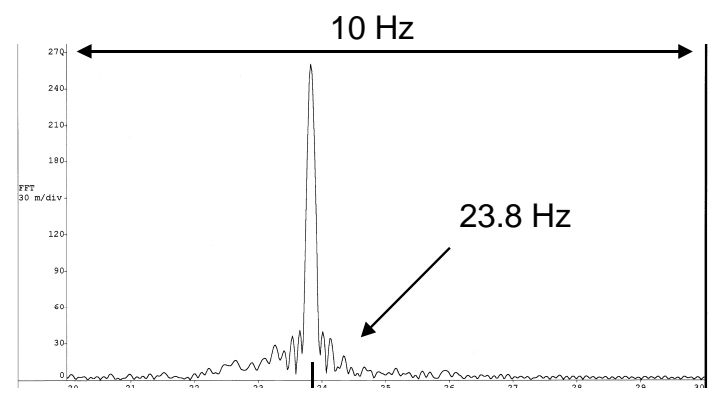

Fig. 3. Fourier analysis of EZ3 torque sensor signal

\section{B. Dynamic performance of the exciter system}

For the modal vibration tests performed, the output power of the thyristor controlled DC circuit was minimized in operating the circuit at small DC output current, $I_{D C}=450 \mathrm{~A}$. This is the smallest current level at which dynamic tests can be performed because the thyristor converter modules of the IPP experimental power supply have nominal (short-time) currents above $20 \mathrm{kA}$ (no sufficient resolution of the current controller for a current modulation with too small amplitude). Fig. 4 shows the excitation of a torsional resonance in the shaft assembly of flywheel generator EZ4 (220 MVA, 600 MJ) by means of a torsional exciter circuit as shown in Fig. 1. The first natural frequency of the EZ4 shaft assembly is $26 \mathrm{~Hz}$. This value and the mechanical characteristics of the EZ4 shaft assembly are similar to the ones of the EZ3 shaft system. The first curve shows the inductor current $i_{L}$ which is modulated with a frequency of $26 \mathrm{~Hz}$. $\mathrm{u}_{\mathrm{L}}$ is the thyristor converter output voltage and $\mathrm{P}_{\mathrm{L}}$ is the active power generated by the dynamically controlled DC circuit. $\mathrm{P}_{\mathrm{L}}$ corresponds to $P_{\text {dist }}$ in Fig. 1. The lowest curve is the torque measured at the EZ4 shaft assembly at a location between the generator rotor and the flywheel. Due to the low natural damping of the rotating steel structure (see frequency spectrum of the torque sensor signal shown in Fig. 3), the oscillating active power causes a slow but constant increase of the measured torque. In order to perform modal vibration tests on site, a compact (mobile) exciter system is necessary. The nominal (continuous) current of such a system should not be higher than 100 A so that standard (air-cooled) power electronic devices can be applied. Since the external pulsating torque must only be applied for a few seconds, it is sufficient to design the electrical circuit generating the oscillating power only for a short-time delivery of active power. That way, the excitation of a torsional resonance as shown in Fig. 4 can be achieved with a thyristor converter, transformer and inductor being designed for a nominal (continuous) current of $120 \mathrm{~A}$ (and a short-time current of $600 \mathrm{~A}$ ).

\section{Parametrical study: Excitation of the first torsional mode in a rigid rotor shaft system}

In an earlier performed study, a numerical simulation model of the arrangement shown in Fig. 1 was derived and the results from numerical simulations were validated by measurement results at a DC current level of $5 \mathrm{kA}$ [7]. The numerical investigations require a detailed model so that the non-linear properties of the synchronous machine, the thyristor converter and the control system can be considered. Since no experiments could be performed with DC currents smaller than the one shown in Fig. 4, investigations with small DC currents were performed by means of the validated numerical simulation model derived for generator EZ3 [7]. A typical simulation result is shown in Fig. 5. The simulations were performed assuming a lower DC component of $\mathrm{i}_{\mathrm{L}}$ than in Fig. 4, but 


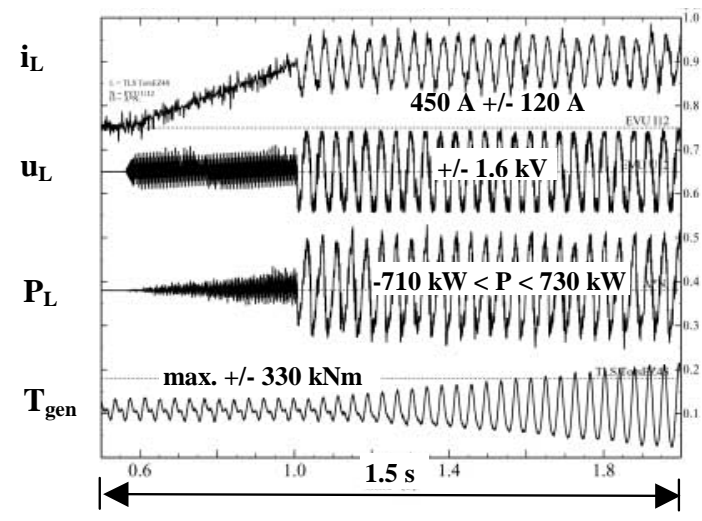

Fig. 4. Measured excitation of a torsional oscillation in the shaft system of flywheel generator EZ4

a slightly increased converter output voltage which is modulated with a frequency of $24 \mathrm{~Hz}$ and an amplitude $\hat{\mathrm{u}}_{L}$ $=1.7 \mathrm{kV}\left(\mathrm{U}_{\mathrm{DC}}=200 \mathrm{~V}\right)$. The high $\mathrm{DC}$ component $\mathrm{U}_{\mathrm{DC}}$ can be explained by the type of inductor used in the simulations. Since the inductor being utilized during the tests at IPP is not of a standard type (a standard inductor would have a much higher ohmic resistance than the one shown in Fig. 1), an inductor with an inductance $L=100$ $\mathrm{mH}$ and a resistance $\mathrm{R}=1 \Omega$ was used in the simulations. Despite of the low current level and the fact that the power circuit of the simulated torsional exciter is based on standard components, it can be seen in Fig. 5 that a dynamic response of the shaft system can be achieved which is comparable to the one shown in Fig. 4. Even higher torques could be applied in utilizing a converter module with higher no-load voltage, e. g. $U_{\mathrm{di} 0}=3 \mathrm{kV}$.

Due to the sharp resonance characteristic of the rigid shaft system, small changes to the excitation frequency can cause significant changes in the measured torque. If only small torques are generated, the torque signal resulting from the electro-mechanical excitation can be superposed by relatively high disturbance signals which make it difficult to achieve accurate measurement results - see lowest curve in Fig. 4. Therefore, the simulation model of the set-up shown in Fig. 1 is used to demonstrate the sensitivity of the EZ3 shaft system to a torsional excitation with a frequency near the first natural frequency. Instead of showing the sensitivity in the time domain, the rotordynamics can be better visualized in displaying locus diagrams of simulated electromechanical excitations, such as the one shown in Fig. 5. In the locus diagram it is most advantageous to plot the torque generated in the shaft system versus a torsional exciter variable being $90^{\circ}$ phase shifted, such as the DC inductor voltage $\mathrm{u}_{\mathrm{L}}$. The optimum excitation is achieved if these two signals remain phase shifted with exactly $90^{\circ}$ during the whole excitation process. Due to the operation of the six-pulse thyristor converter, $\mathrm{u}_{\mathrm{L}}$ is superposed by a sixth harmonic which can be recognized in Fig. 5. Therefore, instead of using $u_{L}$ in the locus diagram, the reference value for the thyristor converter output voltage,
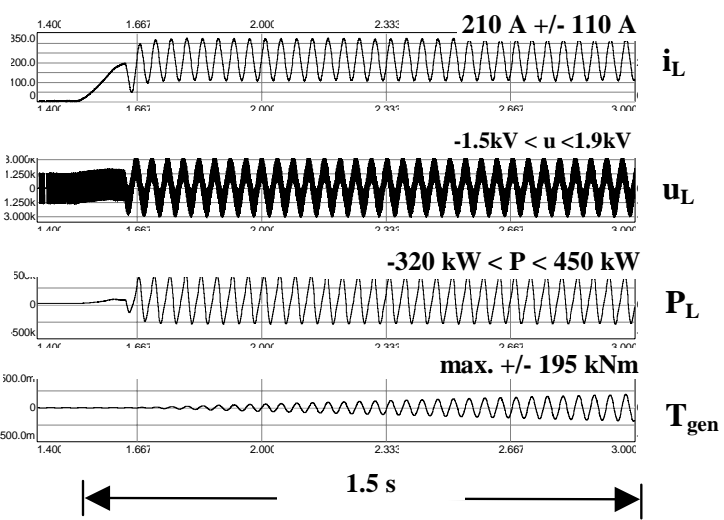

Fig. 5. Simulated excitation of a torsional oscillation in the shaft system of flywheel generator EZ3

$\mathrm{u}_{\mathrm{L}, \mathrm{ref}}$ is used. $\mathrm{u}_{\mathrm{L}, \mathrm{ref}}$ corresponds to the fundamental component of $u_{L}$ which represents the time function of the externally applied torque, see Equations (5) and (6). Fig. 6 shows the resulting locus diagram of the torsional excitation shown in Fig. 5. In order to show the sensitivity of the shaft system to small changes of the fundamental frequency $\Omega$ of such an exciter system, the locus curves in Figs. 7 and 8 show an excitation with the same system parameters but an excitation frequency which differs by $+/-0.3 \mathrm{~Hz}$. The $y$-axis reflects the strong influence of the excitation frequency on the maximum achievable torque. The influence shown in Figs. 6-8 is in good agreement with the measurement result shown in Fig. 3. If an excitation frequency of $23.845 \mathrm{~Hz}$ was chosen, a completely axis-symmetrical locus diagram could be achieved and a torque value of $200 \mathrm{kNm}$ could be reached after 1.5 seconds of torsional excitation. In

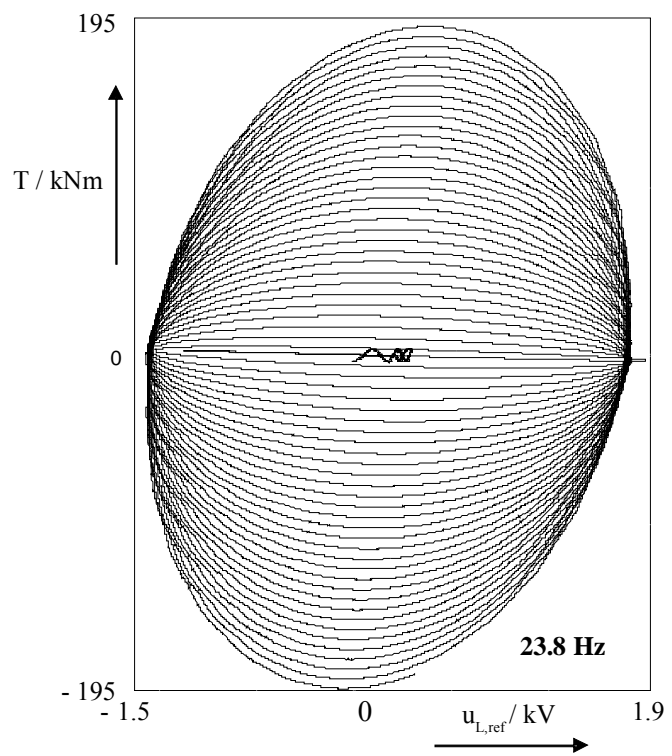

Fig. 6. Locus diagram corresponding to Fig. 5: Plot of the generated torque versus the torsional exciter voltage (exciter frequency: $23.8 \mathrm{~Hz}$ ) 


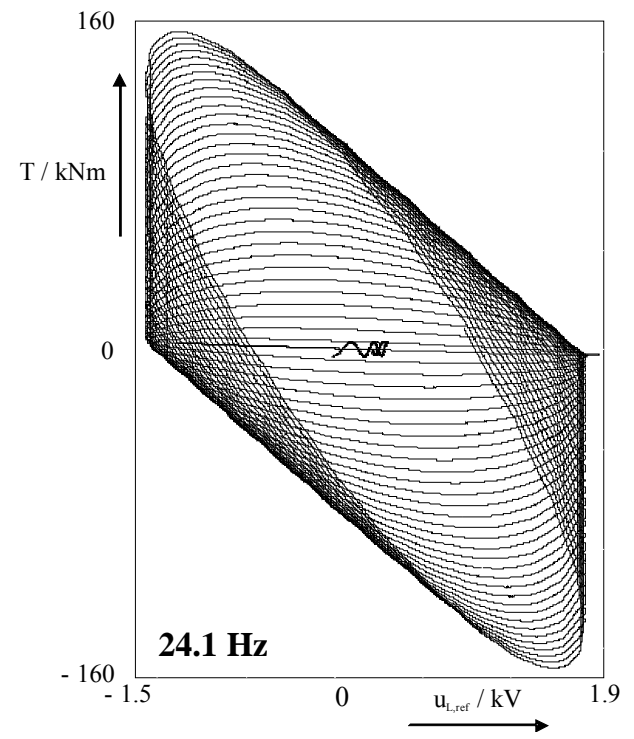

Fig. 7. Plot of the generated torque versus the torsional exciter voltage (exciter frequency: $24.1 \mathrm{~Hz}$ )

contrast to this, a torque value of $160 \mathrm{kNm}(135 \mathrm{kNm})$ cannot be exceeded if an excitation frequency of $24.1 \mathrm{~Hz}$ $(23.5 \mathrm{~Hz})$ is chosen. The locus diagrams in Figs. 6 and 7 show that these maximum values do not depend on the duration of the excitation.

\section{A MOBILE TORSIONAL EXCITER FOR MODAL VIBRATION TESTS}

Drive systems of large rotor shaft assemblies are usually not designed to generate an oscillating active power with a frequency sufficiently high for modal vibration tests. If an existing drive system could fulfill this

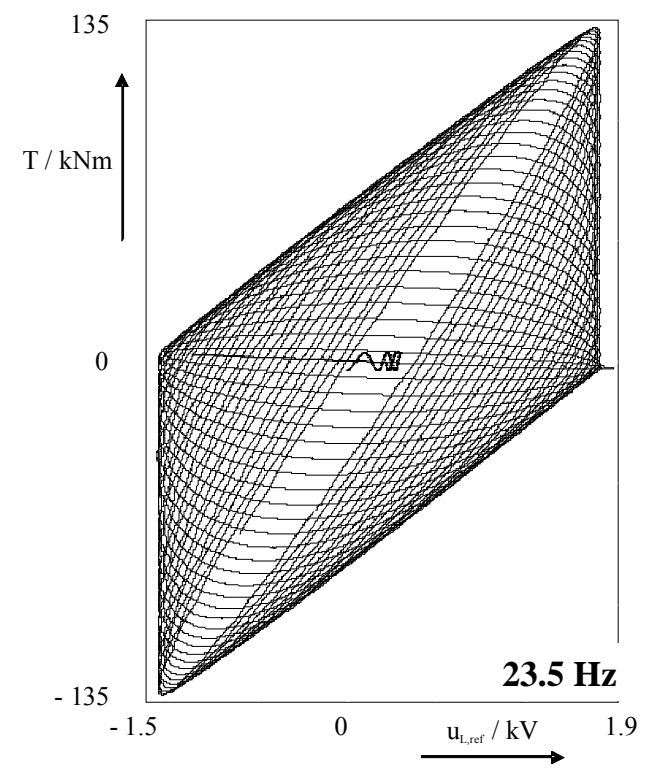

Fig. 8. Plot of the generated torque versus the torsional exciter voltage (exciter frequency: $23.5 \mathrm{~Hz}$ ) requirement, e. g. in case of an IGBT converter supplying a synchronous motor, it would cause much engineering effort to implement a dynamic control scheme for torsional vibration tests and to install all protection devices required for performing dynamic tests with a drive system which has been designed to operate at nominal power of the electrical machine. Therefore, a separate electromagnetic exciter system is more advantageous. It can be designed to operate at low power and could be arranged as a modular, compact unit for flexible use in different applications involving large electrical machinery, e. g. in power plants, wind-electric power stations, industrial facilities, or marine drive systems. The results presented in Section III are based on rotor systems with a simple structure. But a continuous torsional exciter for tests on site would be most advantageous in complex modal testing [5]. The possibility to apply this type of exciter to multi-mass systems has already been demonstrated [10]. An important advantage of the proposed torsional exciter system is that modal vibration tests can be performed without accessing the rotor system, i. e. such tests could be performed during normal operation of the test object. In order to avoid nuisance interactions with other equipment during the tests, they must be performed at low power level, e. g. less than one percent of the nominal power of the electrical machine. Then, a relatively long excitation period is required in order to achieve accurate measurement results. If the exciter is applied in the case of highly coupled rotating structures, like it is the case for different turbine generators in a power plant, precautions have to be taken in order to avoid that a rotating structure not being monitored is subjected to a relevant torsional stress.

As an example for a long lasting excitation with well defined frequencies, Figs. 9 and 10 show measurement results performed at generator EZ4 with an excitation power of less than 1 MW (EZ4 rating: 220 MVA). Considering that rotor systems with unknown oscillatory characteristics must be excited with even lower power than it is shown in Figs. 9 and 10, modal tests based on this exciter method can be time consuming, especially in case of shaft systems featuring sharp resonance points. But the application of a mobile electromagnetic torsional exciter system could be automated, as shown in Fig. 11. After connecting the power circuit of the automated torsional exciter to the three-phase system of the synchronous machine (turbine generator), the modal vibration test can be automatically executed by means of a programmable control circuit. The user can define a frequency range (e. g. $30 \mathrm{~Hz} \leq \Omega \leq 80 \mathrm{~Hz}$ ), a frequency step width (e. g. $\Delta \Omega=0.2 \mathrm{~Hz}$ ) and a threshold for the maximum allowed torque. During the testing, long lasting exciter pulses are sequentially applied, similar to the ones shown in Figs. 9 and 10. The difference in frequency between consecutive pulses is $\Delta \Omega$. If the pre-defined threshold for the measured torque is reached, the corresponding pulse is terminated and the values achieved 
are stored on disk. In the range of critical frequencies modal vibration tests with a smaller frequency step width can be performed in order to determine the oscillatory characteristics of the rotating structure more precisely. Especially the first test sequences being performed at low power could be automatically controlled. One type of

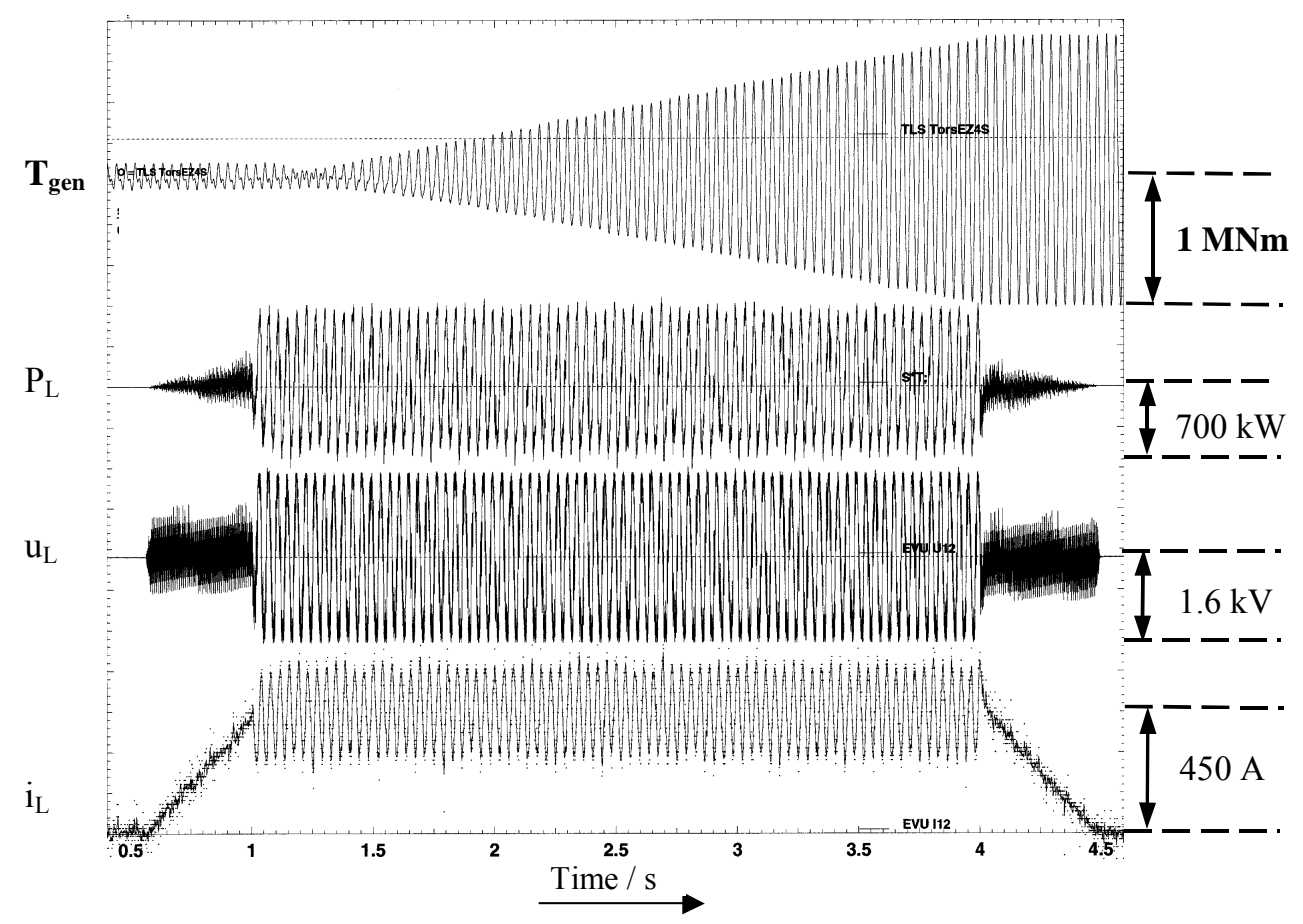

Fig. 9. Measured torsional excitation of generator EZ4 with a frequency of $26 \mathrm{~Hz}$

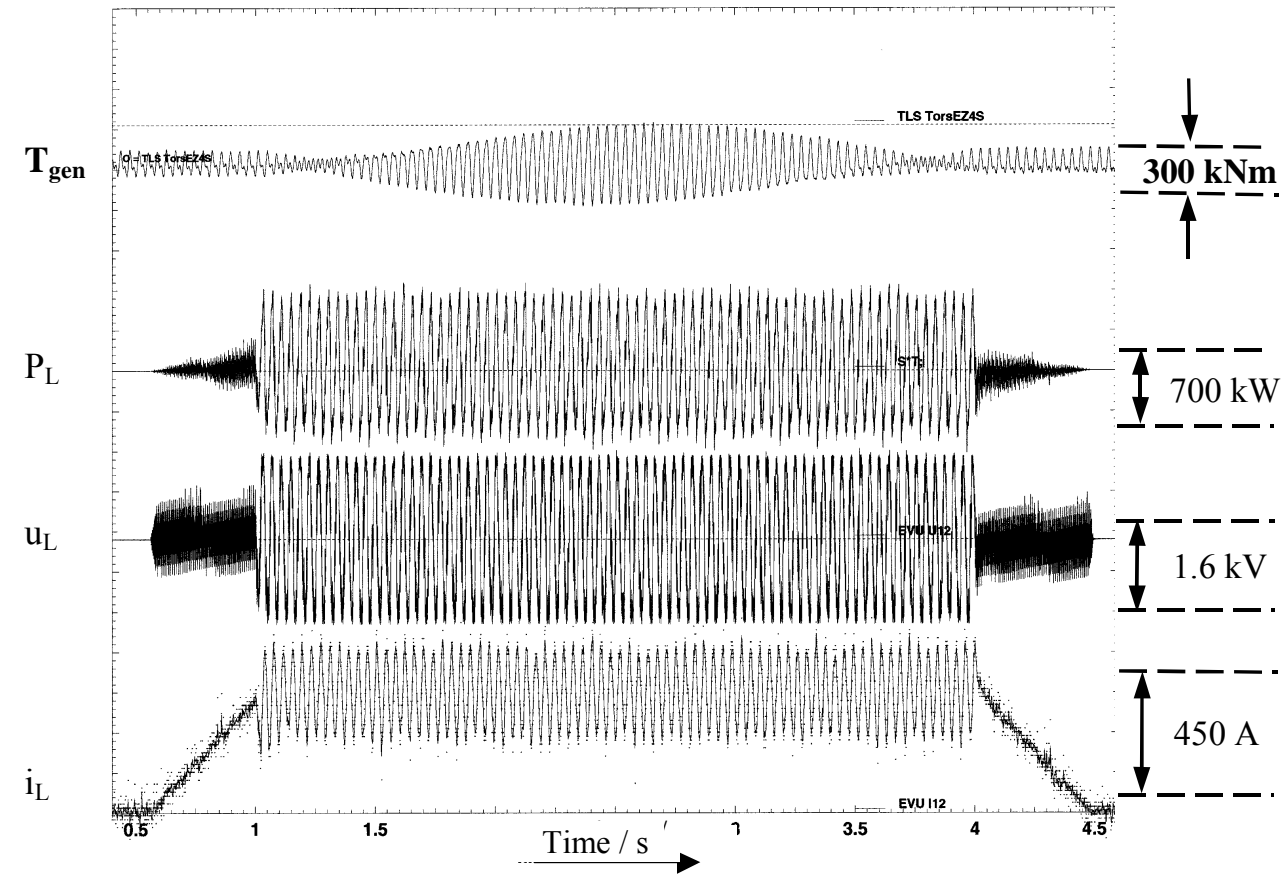

Fig. 10. Measured torsional excitation of generator EZ4 with a frequency of $25.667 \mathrm{~Hz}$ 


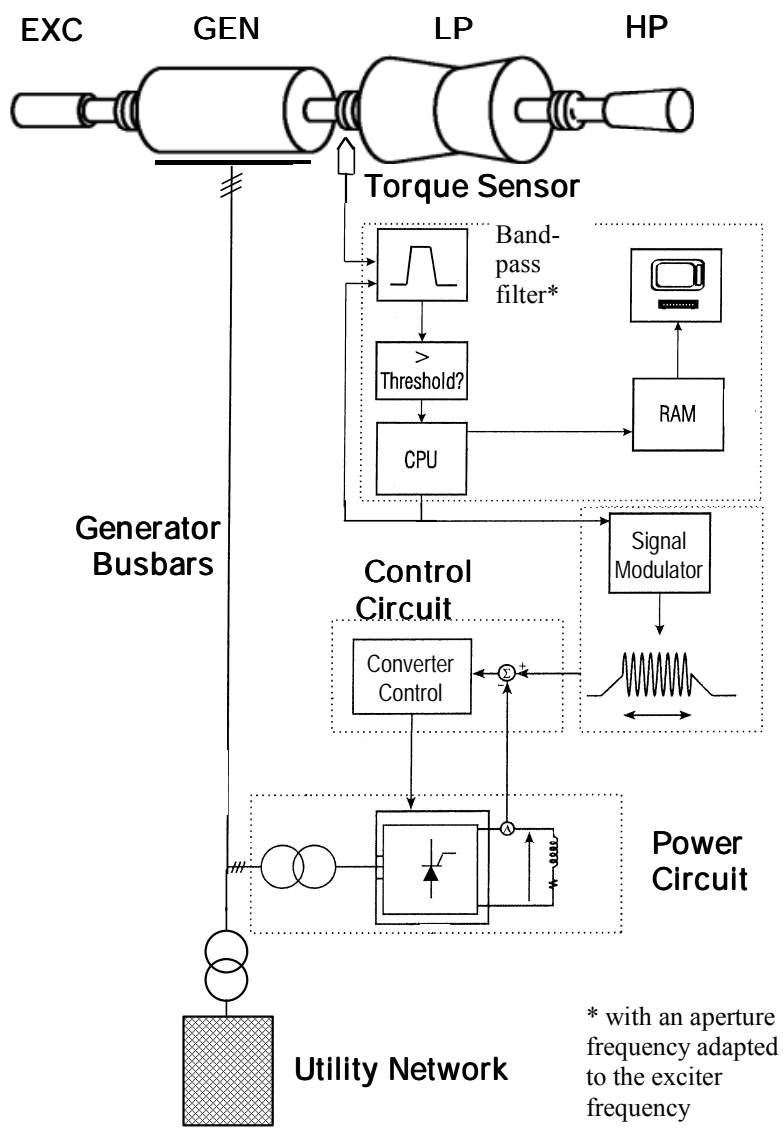

Fig. 11: Schematic of an automated torsional exciter for modal vibration testing of a turbine-generator

design of the torsional exciter could be applied to many different applications if the transformer of the power circuit is designed as a matching transformer. Since the mobile exciter system can be applied on site and the rotating machinery can be operating during the tests, the automated test procedure may be an attractive alternative to conventional test methods.

\section{CONCLUSION}

A torsional exciter for on-site testing of large rotating machinery has been developed and applied to the rotor shaft systems of two large flywheel generators belonging to IPP's experimental power supply. The power circuit of the excitation device is based on a feedback controlled buffer storage of magnetic energy being loaded and unloaded by means of transforming rotational energy from the rotating shaft assembly. Because of this design feature an external power source is not required and the torsional exciter can be designed as a compact (mobile) system for universal application to various shaft assemblies featuring a three-phase motor or generator. Depending on the design of the power circuit, this type of device can be used to generate a continuous torque with adjustable frequency (up to hundreds of hertz) and amplitude (corresponding to a power in the range of kilowatts or megawatts). Since this exciter needs no fixture to the rotating shaft, it can be applied to shaft systems rotating with high speed or shaft systems which must stay in operation while the vibration testing is performed. This requires test runs with low power which may be time consuming but can be performed by means of an automated torsional exciter system. Such a system can sequentially generate long lasting exciter pulses being followed by a cool down period of the power circuitry.

This type of mobile exciter system can also be applied for acceptance testing of new rotating machinery on site (for instance in wind power stations). In this application a defined torque (ranging from tens of kilonewtonmeters to meganewtonmeters) could be generated at critical locations of the rotating shaft system, the torque representing an overload which may occur under extreme operating conditions. High torque values can be generated at various locations of the rigid rotor shaft system in exciting the system with low power but a frequency corresponding to various natural modes of oscillation.

\section{ACKNOWLEDGMENT}

The author would like to thank A. M. Miri (University of Karlsruhe) for his continuous valuable contributions. The author is also grateful to all members of the ASDEX Upgrade team and their contributions to this work.

\section{REFERENCES}

[1] A. M. Dudley, Electric Vibration Generator, US Patent 2,384,987, Westinghouse Electric Corporation, 1945.

[2] C. Teng, D. D. Reynolds, Methods for Resolving Fan/Motor Vibration Problems in Air-Conditioning Units: Part III Experimental Procedures for Investigating Torsional Vibration, ASHRAE Transactions v 104 n Pt 1A, ASHRAE, Atlanta, GA, USA, 1998, pp. 255-262.

[3] G. S. Zobrist et al., Torsional Exciter for a Rotating Structure, US Patent 4,283,957, Zonic Corporation, 1981.

[4] S. H. Crandall, Rotordynamics, p1-44 in Nonlinear Dynamics and Stochastic Mechanics, CRC Press, Boca Raton, 1995.

[5] Y.-D. Joh and C.-W. Lee, Excitation Methods and Modal Parameter Identification in Complex Modal Testing of Rotating Machinery, Modal Analysis: the International Journal of Analytical and Experimental Modal Analysis v 8 n 3, July 1993 , pp. 179-203.

[6] K. Kupfmüller, Einführung in die theoretische Elektrotechnik, Springer Verlag, 9. Auflage, Berlin, 1968.

[7] C. Sihler, A. M. Miri, A. Harada and ASDEX Upgrade Team: Damping of Torsional Resonances in Generator Shafts Using a Feedback Controlled Buffer Storage of Magnetic Energy. In: 5th International Conference on Power Systems Transients, New Orleans, LA 2003, 6b-3. Available: http://www.ipst.org/TechPapers/2003/IPST03Paper6b3.pdf

[8] G. Möltgen, Netzgeführte Stromrichter mit Thyristoren, Siemens AG, Berlin, München, 1967.

[9] P. Lang, S. Kulig, product: "torsional stress analyzer", 2001 [Online]. Available: http://www.itwm.fhg.de.

[10] C. Sihler, Suppression of Torsional Vibrations in Rotor Shaft Systems by a Thyristor Controlled Device, Proceedings of $35^{\text {th }}$ Annual IEEE Power Electronics Specialists Conference, Aachen, Germany, June 2004, pp. 1424-1430. 